\title{
ACTIVIDADES Y TERAPIA ASISTIDA POR ANIMALES DESDE LA MIRADA DEL MODELO DE OCUPACIÓN HUMANA
}

\author{
ACTIVITIES AND ANIMAL ASSISTED THERAPY FROM THE PERSPECTIVE OF THE \\ MODEL OF HUMAN OCCUPATION
}

\section{Julie Agatha Vásquez Duque ${ }^{1}$}

\section{RESUMEN}

Hace algunos años, las Actividades Asistidas con Animales (AAA) y Terapia Asistida por Animales (TAA) han comenzado a formar parte de las intervenciones en Terapia Ocupacional, una práctica que sin duda puede fundamentar y enriquecer la teoría y la sistematización de las intervenciones.

En el presente ensayo, la autora comparte desde el Modelo de Ocupación Humana, parte de la experiencia que ha tenido incluyendo animales como instrumento de intervención en salud. Este comienza con una breve descripción de conceptos, historia y consideraciones en relación a las sesiones de terapia que involucran animales como parte de la intervención terapéutica; continua con los conceptos básicos del Modelo de Ocupación Humana, para luego explicar a través de un ejemplo práctico cómo la Terapia Asistida por Animales puede influir en los procesos volitivos, habituación, capacidad de desempeño y participación ocupacional de las personas, concluyendo finalmente que la importancia de la experiencia volitiva es fundamental en este tipo de intervenciones.
\end{abstract}

\section{PALABRAS CLAVES}

Actividades y Terapia Asistida por Animales, Modelo de Ocupación Humana

\begin{abstract}
A few years ago, Animal Assisted Activities (AAA) and Animal Assisted Therapy (AAT) have begun to be part of interventions in occupational therapy, a practice that can surely enrich the theory and subsequent interventions.

In the present essay, the author share from the Model of Human Occupation, part of the experience she had in including animals as a tool of health intervention. This paper begins with a brief description of concepts, history and considerations regarding therapy sessions involving animals as part of therapeutic intervention, continuing with the basic concepts of the Model of Human Occupation, and then explaining through a practical example how the Animal Assisted Therapy can influence volitional processes, habituation, performance capacity and occupational participation of people, finally concluding that the importance of volitional experience is vital in this type of intervention.
\end{abstract}

\section{KEYWORDS}

Activities and Animal Assisted Therapy, Model of Human Occupation.

1 Terapeuta Ocupacional, Licenciada en Ciencias de la Ocupación Humana. Presidenta Corporación de Adiestramiento Canino para discapacitados “Dos Amigos”. E mail: info@dosamigos.cl 


\section{INTRODUCCIÓN}

La tenencia y cuidado de animales forma parte de la ocupación humana desde épocas ancestrales con fines de alimentación, caza, abrigo, cuidado de ganado, y sin duda como compañía; sin embargo, los primeros registros de la utilización de animales en ambientes terapéuticos con objetivos específicos se encuentran en 1953, cuando el Psiquiatra Boris Levinson atendía a un niño que había pasado por un largo proceso terapéutico sin éxito, y con crecientes síntomas de retraimiento que ameritaban hospitalización. Accidentalmente, ingresó a la consulta su perro Jingles, el niño estableció contacto con el perro y posteriormente expresó su deseo de volver a jugar con él. Desde ese día Levinson utilizó a su perro en todas las sesiones con el niño, logrando involucrarse en el juego y crear un vínculo que posibilitó el abordaje terapéutico (1).

Desde entonces se han realizado estudios que comprueban los beneficios a nivel cardiovascular, psicológico y psicosocial asociados a la tenencia de animales, entre los cuales se puede mencionar: disminución de la presión arterial y niveles de colesterol, disminución del estrés y de la depresión, aumento de la motivación, mejoría en la socialización y disminución de la sensación de soledad, entre otros (2).

Considerando los beneficios que aporta el contacto con animales y que la mayoría de los clientes de Terapia Ocupacional son propietarios de mascotas y cada uno tiene, o podría tener responsabilidad en el cuidado de ellos, es que algunos Terapeutas Ocupacionales han incorporado animales como parte de su método de intervención obteniendo resultados interesantes en las sesiones de tratamiento y en las rutinas diarias del usuario.

Lamentablemente, este tipo de intervenciones tienden a ser vistas como "poco profesionales", lo que probablemente puede deberse a la escasa sistematización de estas experiencias. Con el presente ensayo descrito desde el punto de vista del Modelo de Ocupación Humana, la autora pretende aportar al posicionamiento de la Terapia Asistida con Animales como una disciplina seria que puede ser objeto de investigación científica y herramienta terapéutica eficiente. Esto se ha ejemplificado en la historia ficticia de un niño voluntario, la que incorpora procesos observados tanto en voluntarios como en beneficiarios de este tipo de intervenciones; considerando que una persona puede asumir ambos roles en forma simultánea, mejorando su desempeño ocupacional a través del servicio a los demás con trabajo voluntario.

\section{Marco Conceptual}

Con alguna probabilidad, el lector se pregunte qué significa la Terapia Asistida por Animales. Según la definición de Delta Society, "Terapia Asistida por Animales (TAA) es una intervención dirigida a objetivos terapéuticos, en la cual el encuentro con el animal tiene criterios específicos y es parte integral del proceso de tratamiento" (3). En otras palabras, la gran diferencia entre las Actividades Asistidas por Animales (AAA) y la Terapia Asistida por Animales (TAA) tiene relación con la presencia del profesional de salud.

\section{¿Califica el Terapeuta Ocupacional COMO PROFESIONAL IDÓNEO PARA EL DESEMPEÑO DE ESTE ROL?}

Indudablemente, si se piensa que "el desarrollo de estas actividades, posee todas las características que le permiten ser utilizada con éxito como actividad Ocupacional" (4).

La TAA requiere la participación de la persona en algún nivel de funcionamiento, aunque sea sólo el percibir al animal a través de los sentidos de la vista, tacto, olfato y/o audición, reflejando la participación de la persona en sus áreas de desempeño.

Las Actividades Asistidas con Animales están dirigidas a una meta que puede ir desde ejercicios terapéuticos, al juego o esparcimiento con el animal; se relacionan con los intereses de la persona, tanto en relación a la especie animal, como al tipo de actividad a realizar; tienen significado en algún nivel para la persona, ya que para la mayoría de los beneficiarios, los animales están de alguna forma relacionados con la salud y sentimientos de amor, felicidad, armonía y vitalidad más que con rehabilitación. 
La TAA está determinada por el juicio profesional del Terapeuta Ocupacional y basada en su conocimiento, ya que si bien es una actividad de libre elección, esta debe ser planificada por el profesional basado en la evaluación previamente realizada, con lo que determinará la forma de realizarla, y los tiempos o las variaciones necesarias, adaptándolas y graduándolas, de acuerdo al nivel de desempeño de la persona; La Terapia Asistida por Animales es instrumento para la prevención de la disfunción y /o mantenimiento o mejoramiento de la función o calidad de vida, lo que ya se ha demostrado científicamente.

\section{El Terapeuta, el Usuario/Paciente, el ANIMAL y el Guía del aNimal}

Un factor importante en este tipo de intervenciones, es la participación de al menos cuatro actores:

El rol del Terapeuta es, al igual que en una sesión tipo, evaluar, planificar las sesiones de acuerdo a los objetivos de tratamiento y reevaluar los progresos. Es de suma importancia que el Terapeuta no asuma el rol de Guía del animal en forma simultánea, pues el que sean personas diferentes permite mejor control sobre la sesión y evita problemas en caso de descompensación del Usuario/ Paciente o descontrol del animal.

El rol del Usuario es participar activamente de las sesiones considerando las indicaciones del profesional a cargo.

El rol del guía es participar de las sesiones asegurando que el animal cumpla con las condiciones necesarias para participar en una intervención terapéutica. Cabe destacar que no cualquier animal es apto para ello. La función del guía considera evaluación del carácter del animal, (sociabilidad, sumisión, nivel de tolerancia a la manipulación/dolor, tolerancia a la novedad y diversidad, nivel de actividad), adiestramiento básico (obediencia en el llamado, posición sentado, echado, quieto y caminando juntos) y específico (según los requerimientos para la sesión planificada) y cuidados de salud e higiene (todo animal debe estar sano, vacunado, desparasitado, bañado y con uñas cortas).

El rol del Animal es participar de los adiestramientos y aprender comandos y ejercicios necesarios para la actividad, en la que obedeciendo los comandos que su adiestrador le indica, motiva al usuario a realizar la actividad planificada por el Terapeuta.

\section{Desde la MIRADA DEl Modelo de OCUPACIÓN HUMANA}

El presente ensayo describe los efectos de la Terapia Asistida con Animales desde el Modelo de Ocupación Humana (моно). Un componente central de la ocupación humana incorporado por este modelo se refiere a la Volición o motivación por el hacer. La Volición se manifiesta través de la vida en el Proceso volitivo o ciclo que se despliega en el desarrollo de la causalidad personal (sentido de eficacia personal), los valores (sentido de importancia/significancia) e intereses (sentido de placer) que al interactuar generan las elecciones ocupacionales. El моно define este proceso como "Ios pensamientos y sentimientos volitivos que ocurren en el tiempo a medida que las personas experimentan, interpretan, anticipan y eligen las ocupaciones"(5). En este proceso, la experiencia se refiere a "pensamientos y sentimientos inmediatos que emergen en medio del desempeño" (5); la interpretación se refiere a "el recuerdo y la reflexión sobre el desempeño en términos de su impacto en el sentido de placer, de eficacia y significado para la propia persona y su mundo" (5). Finalmente, la anticipación se refiere a "el proceso de notar potenciales o expectativas para la acción y reaccionar ante ellos" (5).

Este proceso conduce a tomar decisiones a corto y largo plazo: Elecciones de Actividad "decisiones deliberadas y a corto plazo para emprender y abandonar actividades ocupacionales" (6) y Elecciones de Ocupación "compromisos deliberados para entrar en un rol ocupacional, adquirir un nuevo hábito o emprender un proyecto personal" (6).

Las elecciones de ocupación implican hacer un cambio en la Habituación, el "Patrón semi autónomo de comportamiento, organizado en concordancia con nuestros hábitats temporales, físicos y sociales familiares" (7)(8) reflejada en el ejercicio de los hábitos y los roles. Los Hábitos corresponden a las "tendencias adquiridas para responder y desempeñarnos en ciertas formas constantes en ambientes o situaciones familiares" $(7)(8)$. 
Con el tiempo, las elecciones de ocupación y su desempeño en contextos ambientales conducen por una parte, al desarrollo de Roles internalizados o "Incorporación de una posición definida social y/o personalmente y un conjunto relacionando de actitudes y comportamientos" (7)(8) y por otra a mejorar la Capacidad de desempeño "la Capacidad para hacer cosas provista por la condición de los componentes físicos y mentales objetivos subyacentes, acompañada por la experiencia subjetiva correspondiente" (9), lo que retroalimenta el proceso volitivo.

Si bien la Terapia Asistida por Animales puede ser analizada, descrita e investigada desde diferentes marcos de referencia, este artículo realizará una descripción desde el Modelo de Ocupación Humana por ser este uno de los marcos propios de la Terapia Ocupacional, que aborda no sólo habituación y capacidad de desempeño, sino también aspectos emocionales/espirituales que se expresan en la volición y que sin duda se pueden observar claramente tanto en el desarrollo de las sesiones como en el proceso terapéutico en Terapia Asistida por Animales.

\section{Ejemplo de caso}

La autora se inició en Terapia Asistida con Animales el año 2004 en la "Corporación Dos Amigos", institución que trabaja en base al voluntariado con personas de diferentes edades, ocupaciones, niveles socio económicos y capacidades/ discapacidades. Si bien las diferencias entre las personas dan cuenta de una marcada heterogeneidad, los procesos observados durante estos años son altamente parecidos. De este modo, nuestro objetivo es reflexionar a partir del caso ficticio de una niña que se involucra en TAA y cuyo propósito está relacionado con inserción social.

\section{PRIMER DÍA}

“iHe estado cerca de animales! Son suaves, tiernos y me hacen sentir acompañada y protegida. ¡Los animales que he conocido me gustan tanto que quisiera verlos más seguido, ojala tenerlos en casa! Mi mamá dice que dan mucho trabajo, pero no importa, me gusta alimentarlos y limpiarlos.

\section{SEGUNDO DÍA}

"Hoy es sábado, puedo cortar unas salchichas para llevarles de premio. ¡Sé que les encantan!"

\section{TERCER DÍA}

"Mi papá dice que hoy podemos ir al cine, pero yo le dije que mejor mañana porque hoy quiero estar con los animales"

\section{SEGUNDO MES}

"Hoy pidieron voluntarios para ser familia adoptiva de perros de servicio, lo conversamos en familia y jvamos a participar! Tendré que organizarme muy bien para enseñarle buenos hábitos, ir a clase una vez por semana y adiestrarlo todos los días."

\section{QUINTO MES}

"Hoy llegué del colegio y como de costumbre me dirigí a alimentar a Nanu y limpiar el patio. Cuando no vino a mí, me acordé que estaba en la clínica para su esterilización".

\section{SEXTO MES}

“En el colegio tenemos que hacer una disertación. Yo escogí hablar sobre los perros de servicio porque me encanta servoluntaria, además, mis compañeros saben que adiestro a mi perro y siempre me preguntan cosas".

\section{SÉPTIMO MES}

“iLa disertación me salió muy bien! Antes me costaba hablar en público, pero desde que soy voluntaria todos me preguntan cosas en la calle mientras adiestro a Nanu, ahora es más fácil decir lo que sé. Me puse de pie e incluso hice demostraciones"

\section{Discusión}

Mucho se ha dicho sobre los animales y los beneficios a la salud humana, otorgándoles incluso cierto misticismo; sin embargo, desde la experiencia de la autora, esta no difiere en gran medida de otro tipo de intervención terapéutica. Muy por el contrario, requiere de la rigurosidad y profesionalismo de quienes la practican. Pero ¿qué hace de este tipo de intervenciones algo exitoso?

Retomando el ejemplo y considerando la importancia del experimentar, probablemente encontremos la respuesta a la anterior pregunta. 
“ie estado cerca de animales! Son suaves, tiernos y me hacen sentir acompañada y protegida". Para esta niña, la primera experiencia con este tipo de animales fue algo agradable al tacto, y evocó un sentimiento de seguridad; lo interpreta como una actividad que presenta alto grado de interés "iLos animales que he conocido me gustan tanto que quisiera verlos más seguido, ojalá tenerlos en casa!" y anticipa un próximo encuentro tan agradable como el primero en el que mostrará iniciativa. "Hoy es sábado, puedo cortar unas salchichas para llevarles de premio. ¡Sé que les encantan!".

He aquí la importancia de las primeras impresiones en Terapia Ocupacional, y específicamente en Terapia Asistida con Animales.

Si logramos ofrecer una actividad motivadora, la anticipación naturalmente llevará a tomar decisiones positivas en relación a esa actividad: Elecciones de actividad.

"Mi papá dice que hoy podemos ir al cine, pero yo le dije que mejor mañana porque hoy quiero estar con los animales".

$\mathrm{Si}$ el proceso ha favorecido un fortalecimiento de la volición, la persona tomará decisiones que impliquen mayor compromiso, lo que se ve reflejado en cambio de hábitos, la adquisición de un nuevo rol o proyectarse a futuro. Elecciones de Ocupación.

"Hoy pidieron voluntarios para ser familia adoptiva de perros de servicio, lo conversamos en familia y ivamos a participar! Tendré que organizarme muy bien para enseñarle buenos hábitos, ir a clase una vez por semana y adiestrarlo todos los días."

Este es el caso de una niña, no obstante hasta el momento hemos omitido si padece de alguna discapacidad. Los animales no discriminan por estado de salud, se comportan de igual manera y todos requieren cuidados de alimentación, higiene, salud y socialización. Hay muchos casos de personas con discapacidad que han decidido adiestrar a su propio perro para que los ayude cuando lo requieran, y hay otros que incluso han decidido enseñar a otros a hacerlo.

Por otro lado, cabe preguntarse si existe diferencia cuando una persona elige participar activamente de su rehabilitación en lugar de sólo asistir por prescripción médica. A nuestro juicio claramente podemos motivar esta decisión con la ayuda de los animales siempre y cuando se realice una evaluación completa, considerando tanto las experiencias, interpretaciones, anticipaciones y decisiones volitivas del pasado, como las motivaciones presentes en torno al tema.

Sin duda, las elecciones influyen directamente en la Habituación. Para desempeñar la Ocupación elegida muchas veces se debe cambiar los Hábitos, pues las elecciones de Ocupación demandan organizarse en rutinas para optimizar el tiempo y lograr cumplir con todas las actividades involucradas, las que con el tiempo llegan a ser incluso automáticas "Hoy llegué del colegio y como de costumbre me dirigí a alimentar a Nanu y limpiar el patio. Cuando no vino a mí, me acordé que estaba en la clínica para su esterilización."

De la misma manera, son las elecciones de ocupación las que llevan a adoptar una posición social, y que en conjunto con los comportamientos o actitudes que se esperan de cada uno, tomará la forma de Rol Internalizado.

"En el colegio tenemos que hacer una disertación. Yo escogí hablar sobre los perros de servicio porque me encanta ser voluntaria, además, mis compañeros saben que adiestro a mi perro y siempre me preguntan cosas".

En la experiencia de la autora, es la habituación la que contribuye a los cambios considerables y estables en el tiempo. Eso no significa perpetuar las terapias, ni siquiera si esta es vivenciada como agradable para el usuario. TAA significa que una vez identificada la necesidad de aumentar la responsabilidad y la complejidad, debemos buscar alternativas relevantes, entre las cuales puede estar el voluntariado o ser propietario de un animal que cumpla las características necesarias según el ambiente en que vive y se desenvuelve la persona.

En la medida que se participe de sesiones estructuradas constantes en el tiempo que desarrollen un sentido de capacidad personal y autoeficacia (10), mejorando así la apreciación de sí mismo, podremos observar un impacto en la capacidad de desempeño.

Esto se refleja en el ejemplo "iLa disertación me salió muy bien! Antes me costaba hablar en público, pero desde que soy voluntaria todos me preguntan cosas en la calle 
ESTRATEGIAS UTILIZANDO ANIMALES COMO HERRAMIENTA TERAPÉUTICA

\begin{tabular}{|c|c|c|}
\hline $\begin{array}{l}\text { Etapas de } \\
\text { Intervención } \\
\text { MOHO }\end{array}$ & Dimensiones & Ejemplos de Abordaje con TAA \\
\hline \multirow[t]{4}{*}{ Exploración } & Elección & $\begin{array}{l}\text { Para esto se comienza mostrando los "trucos" que los animales saben hacer. Al } \\
\text { principio desde lejos, y en la medida que la persona se siente segura, ir acercán- } \\
\text { dose hasta que sea el usuario quien manifieste o acepte ser quien da los coman- } \\
\text { dos al animal. }\end{array}$ \\
\hline & Descubrimiento & $\begin{array}{l}\text { Con el juego de obediencia se busca que la persona pueda descubrir que también } \\
\text { puede influir en las acciones del animal, darse cuenta de las habilidades que des- } \\
\text { plegó en la actividad y no conocía o creía perdidas. }\end{array}$ \\
\hline & $\begin{array}{l}\text { Absolución de } \\
\text { Fracaso }\end{array}$ & $\begin{array}{l}\text { En esta etapa, el guía puede dar el comando con lenguaje corporal acompañando } \\
\text { el lenguaje del usuario, a fin de que el usuario reciba respuesta a su interacción. }\end{array}$ \\
\hline & Diversión-placer & $\begin{array}{l}\text { Una vez que el usuario está involucrado en la actividad y muestra mayor confian- } \\
\text { za y cercanía, el guía disminuye el apoyo con comandos corporales y se permite } \\
\text { errores por parte del animal, los que son tomados como algo natural. Se invita al } \\
\text { usuario a volver a intentarlo. }\end{array}$ \\
\hline
\end{tabular}

Ausencia de límites En esta etapa es importante respetar los tiempos y distancia del usuario, permitiénde tiempo dole que en algunos casos sólo observe desde lejos por unos minutos.

Si en el caso contrario, el usuario no desea dar término a la actividad, es importante clarificar la necesidad de descanso del animal y aprovechar la oportunidad para favorecer la continuidad de las próximas sesiones.

Competencia Enseñanza directa El Terapeuta Ocupacional organiza diferentes actividades, las que pueden estar de habilidades planteadas desde distintos marcos de referencia, según la necesidad. El animal puede servir de motivador para trabajar prehensiones, marcha, organización espacial, memoria y concentración, entre otros.

Orientación Si el usuario manifiesta el deseo de adquirir una mascota o un perro de asistencia, el terapeuta orienta en relación a las demandas que implica la adquisición del nuevo rol (proveedor de cuidados, propietario, adiestrador) y orientar respecto a los pasos a seguir y las personas que pueden apoyar en la elección del animal según las características y necesidades del usuario y adiestramiento básico.

Participación en situaciones reales de la vida diaria

Manejo Ambiental

Sesiones de seguimiento con Terapeuta Ocupacional
Se puede abordar considerando las tareas que demanda tener una mascota (alimentación, aseo, ejercicio y recreación) El terapeuta evalúa el desempeño de la actividad y da indicaciones para el logro. También en esta dimensión se considera la capacitación en el desempeño de las AVDı con el apoyo de los perros de asistencia.

El T.O. apoya al usuario para que logre desempeñar en forma más expedita su nuevo rol a través de adaptaciones como engrosamiento de cepillos y correas o cambio altura de plato de comida.

En caso que la persona haya elegido tener un perro de asistencia, es importante determinar en qué actividades el perro va a prestar apoyo y fomentar que el usuario realice por sí mismo las otras actividades, aún cuando el perro esté adiestrado para hacerlo.

Si no es el mismo usuario quien entrena a su perro, se debe mantener un vínculo con el adiestrador del animal para que la enseñanza de comandos sea de acuerdo a las necesidades del usuario.

Participación en grupos de autoayuda
Si el usuario elige adiestrar a su propio animal de asistencia, debe participar de alguna institución que lo apoye en el proceso, ya sea como curso formal o voluntariado. 
mientras adiestro a Nanu, ahora es más fácil decir lo que sé. Me puse de pie e incluso hice demostraciones".

Para la mayoría de las personas, hacer demostraciones es algo fluído, sin mayores complicaciones, sin embargo, para quien tiene dificultad, por ejemplo en el desplazamiento o en las prehensiones, cada nuevo ejercicio que realiza con el animal significa progreso en su capacidad de desempeño. Lograr coordinar, dar la orden al animal, hacer el ejercicio y premiarlo con golosinas requiere práctica y constancia.

Como dice Kielhofner "Las nuevas experiencias pueden alterar el punto de vista de nuestras capacidades. La experiencia puede mostrarnos felizmente que tenemos hasta ahora un talento oculto" (10), "Cuando sabemos que somos capaces y eficaces, estamos dispuestos a actuary generar nuevas evidencias de nuestro talento" (10).

\section{CONCLUSIONES}

La tenencia y cuidado de animales es una actividad ocupacional a la que la mayoría de nuestros usuarios se han enfrentado en algún momento de sus vidas.

Si bien cada experiencia de Terapia Ocupacional es única, la Terapia Asistida por Animales, nos ofrece una gama mucho amplia en este sentido, por contar con seres vivientes como herramienta terapéutica, pues la retroalimentación recibida es constantemente dinámica.

Analizando la Terapia Asistida con Animales desde el punto de vista del Modelo de Ocupación Humana, podemos concluir que ambas disciplinas son totalmente compatibles y complementarias. En TAA, el foco del usuario no está puesto en una parte de su cuerpo o en alguna "deficiencia" que requiera mejorar, sino en la interacción con el animal y logro de la actividad; la persona despliega sus habilidades para la adaptación, recibiendo a través del comportamiento del animal una respuesta inmediata a una acción lograda, lo que es altamente motivante. Esto concuerda totalmente con el concepto de Cuerpo Vivido que el моно considera al momento de abordar la Capacidad de desempeño y que plantea experimentar el cuerpo como sujeto, lo que favorece una experiencia volitiva exitosa, pues un ejercicio, actividad u ocupación realizada con un sentido de competencia y/o logro, constituye en sí el inicio de un nuevo proceso volitivo que prepara a la persona a elegir participar en otras experiencias ocupacionales dentro de otros ambientes en los que no necesariamente hayan animales. Es así como en el desarrollo de la experiencia en el trabajo con animales, personas que han desplazando sus sillas de ruedas para utilizar bastones, han decidido participar de los Juegos Paraolímpicos, han logrado la confianza necesaria para buscary encontrar un trabajo o tomado finalmente la decisión de casarse o tener hijos. Esa es la misma esencia y finalidad de la Terapia Ocupacional.

\section{REFERENCIAS BibLIOGRÁfICAS}

1. Lewinson, B. Pet-oriented child psicotherapy. Springfield, Illinois, Charles C Thomas Publisher LTD, 1997. 37p.

2. Fine A. Handbook on Animal Assisted therapy. Theorical foundations and guidelines for practices. California, Academic Press, 2000. pp. 42-56.

3. Howie, A. Starting a Visiting Animal Group. Washington, Providence health sistem, 2000. 2.

4. Arriaza, C., Perez, M. \& Valdes, A. Impacto de la interacción de personas con animales de compañia: Implicaciones en la salud humana y relación con la Terapia Ocupacional. Revista Chilena de Terapia Ocupacional, Vol. 1:29, 2001

5. Kielhofner, G. Motivos, Patrones y Desempeño de la Ocupación: Conceptos Básicos. En su: Terapia Ocupacional Modelo de Ocupación Humana teoría y aplicación. $3^{\circ}$ Ed. Buenos Aires, Editorial Médica Panamericana, 2004. Pp.15-20.

6. Kielhofner, G. Motivos, Patrones y Desempeño de la Ocupación: Conceptos Básicos. En su: Terapia Ocupacional Modelo de Ocupación Humana teoría y aplicación. $3^{\circ}$ Ed. Buenos Aires, Editorial Médica Panamericana, 2004. P.20

7. Kielhofner, G. Motivos, Patrones y Desempeño de la Ocupación: Conceptos Básicos. En su: Terapia Ocupacional Modelo de Ocupación Humana teoría y aplicación. $3^{\circ}$ Ed. Buenos Aires, Editorial Médica Panamericana, 2004. Pp.22-25

8. Kielhofner, G. Motivos, Patrones y Desempeño de la Ocupación: Conceptos Básicos. En su: Terapia Ocupacional Modelo de Ocupación Humana teoría y aplicación. $3^{\circ}$ Ed. Buenos Aires, Editorial Médica Panamericana, 2004. Pp.15-20

9. Kielhofner, G. Habituación: Patrones de la Ocupación Diaria. En su: Terapia Ocupacional Modelo de Ocupación Humana teoría y aplicación. $3^{\circ}$ Ed. Buenos Aires, Editorial Médica Panamericana, 2004. Pp.71-81

10. Kielhofner, G. Volición. En su: Terapia Ocupacional Modelo de Ocupación Humana teoría y aplicación. $3^{\circ}$ Ed. Buenos Aires, Editorial Médica Panamericana, 2004. Pp. 51-54. 